Classification: Biological Sciences: Biophysics and Computational Biology

\title{
SurA is a "Groove-y" Chaperone That Expands Unfolded Outer Membrane Proteins
}

Dagan C. Marx ${ }^{1}$, Ashlee M. Plummer ${ }^{1 \ddagger}$, Anneliese M. Faustino ${ }^{2}$, Taylor Devlin ${ }^{1}$, Michaela A. Roskopf $^{1}$, Mathis J. Leblanc ${ }^{1}$, Henry J. Lessen ${ }^{1}$, Barbara T. Amann ${ }^{1}$, Patrick J. Fleming ${ }^{1}$, Susan Krueger $^{3}$, Stephen D. Fried ${ }^{2}$, Karen G. Fleming ${ }^{1 *}$

${ }^{1}$ Thomas C. Jenkins Department of Biophysics, Johns Hopkins University, Baltimore, MD

${ }^{2}$ Department of Chemistry, Johns Hopkins University, Baltimore, MD

${ }^{3}$ National Institute of Standards and Technology, Gaithersburg, MD

${ }^{\ddagger}$ Current Address: Department of Cell Biology, Harvard Medical School, Boston, MA

*Corresponding Author: Karen.Fleming@jhu.edu

Keywords: Periplasmic Chaperones, Outer Membrane Protein Biogenesis, Crosslinking Mass Spectrometry, Small-Angle Neutron Scattering, Integrative/Hybrid Structural Biology

Competing interest statement: The authors declare no competing interests.

Data access statement: All raw data and detailed protocols including gel images, SANS profiles, and model creation protocols, are available upon request. The SI Appendix includes detailed protocols and raw data associated with XL-MS experiments.

\footnotetext{
Abbreviations:

SurA $\bullet$ UOMP - non-crosslinked protein-protein complex, SurA-uOMP - crosslinked protein-protein complex uOMP - unfolded outer membrane protein BAM - beta barrel assembly machinery pAF - para-azido phenylalanine pXL-MS - photo-crosslinking mass spectrometry SASD - solvent accessible surface distance
} 
Abstract: The periplasmic chaperone network ensures the biogenesis of bacterial outer membrane proteins (OMPs) and has recently been identified as a promising target for antibiotics. SurA is the most important member of this network both due to its genetic interaction with the $\beta$-barrel assembly machinery complex as well as its ability to prevent unfolded OMP (uOMP) aggregation. Using only binding energy, the mechanism by which SurA carries out these two functions is not well understood. Here we use a combination of photocrosslinking, mass spectrometry, solution scattering, and molecular modeling techniques to elucidate the key structural features that define how SurA solubilizes uOMPs. Our experimental data support a model in which SurA binds UOMPs in a groove formed between the core and P1 domains. This binding event results in a drastic expansion of the rest of the UOMP, which has many biological implications. Using these experimental data as restraints, we adopted an integrative modeling approach to create a sparse ensemble of models of a SurA •uOMP complex. We validated key structural features of the SurA•uOMP ensemble using independent scattering and chemical crosslinking data. Our data suggest that SurA utilizes three distinct binding modes to interact with uOMPs and that more than one SurA can bind a UOMP at a time. This work demonstrates that SurA operates in a distinct fashion compared to other chaperones in the OMP biogenesis network.

Significance Statement: Outer membrane proteins play critical roles in bacterial physiology and increasingly are exploited as antibiotic targets. SurA is the most important chaperone in the OMP biogenesis network and is thought to initiate their folding through an interaction with the BAM complex. We observe an unprecedented expansion of unfolded outer membrane proteins when bound to SurA. This expansion suggests a potential mechanism by which SurA can deliver uOMPs to the BAM complex. In addition, this study highlights the use of an integrative/hybrid structural biology approach and emerging methods to map highly heterogeneous structural ensembles such as that of an unfolded protein bound to a chaperone. 


\section{$\underline{\text { Introduction }}$}

Proteins must fold into their native three-dimensional structures to perform their functions. For some proteins this folding process is spontaneous and requires no exogenous factors; however, many proteins - particularly, membrane proteins - are predisposed to populate misfolded states or aggregates that are not functional and can be toxic to the cell (13). To suppress these pathways, chaperone proteins promote efficient protein folding through interactions with nascent, unfolded proteins (termed clients) (4-8).

One chaperone network of particular importance is the outer membrane protein (OMP) biogenesis network in gram-negative bacteria. Following translocation across the inner membrane, this network solubilizes hydrophobic, unfolded OMPs (uOMPs) in the aqueous periplasm and delivers them to the $\beta$-barrel assembly machine (BAM) complex, which catalyzes uOMP folding into the outer membrane (9-13). OMPs play several critical roles in bacterial physiology such as nutrient uptake, lipid remodeling, and efflux (14). Recently, the OMP biogenesis pathway has been exploited as a target for the development of antibiotics against gram-negative bacteria because drugs that compromise essential OMP maturation need only cross the fairly porous outer membrane and not the tightly regulated inner membrane (15-17).

The uOMP biogenesis chaperone network is comprised of three proteins: SurA, which has been shown to be the most important protein in the pathway, as well as Skp and FkpA (1823). SurA handles the majority of the flux of unfolded outer membrane proteins (uOMPs) through the periplasm, and accordingly a $\Delta s u r A$ null-strain induces a pronounced envelope stress response $(9,18,24-29)$. Eight OMPs of varying size (8-26 transmembrane $\beta$-strands) and sequence composition have been identified as SurA clients because their expression is notably decreased in the $\Delta$ surA strain $(14,28,30-32)$.

The mechanism by which SurA binds and solubilizes client uOMPs is currently unknown. The lack of ATP in the periplasm implies that the driving forces for SurA's function must derive from interactions with its clients. Unlike the other members of the OMP biogenesis network that oligomerize to form cages around uOMPs, SurA lacks an obvious cavity to shield uOMPs from the aqueous periplasm and does not readily oligomerize in solution (9). Instead, SurA has 
a modular structure with three distinct domains connected by flexible linkers shown in Figure 1A: a primarily alpha helical "core" domain comprised of portions from the $\mathrm{N}$ - and C-terminal regions, and two peptidyl prolyl isomerase (PPlase) domains (P1 and P2) (33-36). The orientations of the $\mathrm{P} 1$ and $\mathrm{P} 2$ domains relative to the core domain have been shown to be dynamic, though how the SurA conformational ensemble contributes to uOMP binding is unclear $(22,37-39)$. The core domain of SurA is thought to be responsible for the majority of the binding energy to small, eight-stranded uOMPs and alone can complement the $\Delta$ surA strain of $E$. coli $(22,30,40-48)$.

Structural elucidation of a SurA•uOMP conformation is challenging. As discussed above, SurA has been shown to exist in multiple conformations. Moreover, the unfolded nature of client OMPs poses several additional hurdles that have impeded structural studies using classical techniques: uOMPs lack regular secondary structure, are highly dynamic, and are prone to aggregation (49-52). We address these challenges by capitalizing on the power of an integrative/hybrid structural biology approach that combines data from crosslinking, mass spectrometry, and neutron scattering to elucidate structural features of the SurA•uOMP ensemble. By incorporating photo-crosslinking unnatural amino acids throughout SurA, we find SurA sites with the highest crosslinking efficiencies to clients line a groove formed between the core and P1 domains. Contrast-matching small angle neutron scattering (SANS) of a crosslinked complex reveals that a canonical uOMP client is greatly expanded when bound to SurA. Mass spectrometry analysis of the photo-crosslinked SurA-uOMP complexes identifies specific segments on client uOMPs that preferentially interact with the SurA groove.

Using our experimental data as restraints, we created a sparse ensemble of 40 configurations of SurA•uOMP complexes. We validated structural features present in this ensemble with additional chemical crosslinking mass spectrometry and SANS experiments that were not included in model generation. We identified three distinct UOMP binding modes and higher order stoichiometries that were sufficient to explain the data. Overall, our findings provide a structural basis for how SurA solubilizes its UOMP clients and provide a template for how future studies might elucidate the structures of highly dynamic chaperone complexes with unfolded proteins. 


\section{$\underline{\text { Results }}$}

SurA crosslinks preferentially with client uOMPs. We identified regions of SurA involved in binding uOMPs using short-distance crosslinkers across the surface of SurA. To accomplish this, we incorporated the unnatural amino acid, para-azido-Phenylalanine (pAF), at 32 nonconserved, surface-exposed positions on SurA (Figure 1B; see SI Appendix, Fig. S1) using amber suppression (53). pAF is a 'zero-length' crosslinker, because it forms highly reactive intermediates that crosslink rapidly and non-specifically to any residue within 3-4 $\AA$ (though one crosslinking mechanism has been shown to slightly favor reactions with aromatic amino acids) $(54,55)$. Previously applied in biochemical assays, we report here to the best of our knowledge the first application of pAF crosslinking for structural studies $(56,57)$.

Each SurA variant with a single amino acid substituted for pAF (denoted SurA $A_{p A F}$ ) was mixed with one of three uOMPs: clients $\mathrm{uOmpA}_{171}$ (the transmembrane domain of uOmpA, see SI Methods), uOmpX, or a non-client uOmpLA as a negative control $(31,58)$. Samples were exposed to UV light for 5 minutes and crosslinking efficiency was measured by quantitative SDSPAGE (see SI Appendix, Figure S2, Table S1) (59). Crosslinking efficiencies to the two client uOMPs vary dramatically with position on SurA, with the highest efficiency sites all residing on the core and P1 domains (Figures 1B,C). The finding that high-efficiency crosslinking sites for client uOMPs map to the SurA P1 and core domains is consistent with previous experiments that found that removal of the P2 domain did not affect the binding affinity of $u O m p A_{171}$ to SurA (22). In contrast, the non-client UOmpLA showed low crosslinking efficiencies; indeed, only half of the SurA $A_{p A F}$ variants could form crosslinks with uOmpLA at all (see SI Appendix, Figure S3, Table S1). In addition to the high-efficiency crosslinking sites on cognate client uOMPs, we observed lower levels of crosslinking to client UOMPs at other pAF sites across the surface of SurA. The observed differences in crosslinking efficiencies between client and non-client uOMPs indicate that SurA is inherently able to distinguish client UOMP sequences in solution without the aid of other chaperones.

SurA binds uOMPs in a groove between the core and P1 domains. We sought to identify the uOMP binding site on SurA by visualizing the high-efficiency pAF crosslinking sites on known conformations of SurA (see SI Appendix, Table S2)(37, 39). This analysis revealed that residues 
colocalized around a groove that forms between the core and P1 domains in the open conformation (Figure 1D, see SI Appendix, Fig. S4 and S5). In this conformation, both of the PPlase domains are structurally isolated from the core domain (shown in Figure $1 A, B, D$ ).

The uOMP-binding groove is large enough ( $25 \times 25 \times 25 \AA)$ to shield from water the entire length of either a transmembrane $\beta$-strand or $\beta$-hairpin of an UOMP (see SI Appendix, Figure S5A). The walls of the groove are formed by the core and P1 domains, which provide hydrophobic patches surrounded by weakly positively charged regions. The base of the groove contains a long hydrophobic stretch (30 ̊) and is more positively charged than the walls. Interestingly, the regions of the core and $\mathrm{P} 1$ domains outside of the groove are highly negatively charged. The separation of charges on SurA could allow electrostatics to play a role in driving uOMP binding to the groove (see SI Appendix, Figure S5B). In sum, the SurA groove possesses a hybrid chemical nature and size well suited to accommodate the alternating hydrophobic-hydrophilic patterning of uOMP transmembrane domains.

SurA solubilizes $\mathrm{uOmpA}_{171}$ in an expanded conformation. uOMPs are expected to exist in a relatively collapsed, molten globule state in the absence of chaperones (50). This collapsed conformation is maintained when $\mathrm{uOmpA}_{171}$ is bound by the other major chaperone in the uOMP biogenesis pathway, $\operatorname{Skp}(60,61)$. To determine whether the overall size and shape of a uOMP changes when bound to SurA, we measured the hydrodynamic properties using small angle neutron scattering (SANS). SANS reports directly on the radius of gyration $\left(R_{\mathrm{G}}\right)$ and the maximum end-to-end distance $\left(D_{\mathrm{Max}}\right)$ of macromolecules. Moreover, the sample and buffer conditions can be manipulated in a SANS experiment to visualize a selected component within a complex.

We capitalized on this selective contrast feature of SANS and collected the scattering profile of a photo-crosslinked complex composed of protonated SurA $\mathrm{A}_{105, \mathrm{pAF}}$ and perdeuterated $\mathrm{uOmpA}_{171}$ in $30 \% \mathrm{D}_{2} \mathrm{O}$ (Figure $2 \mathrm{~A}$ ). In this experiment, SurA contributes a minor fraction to the scattering contrast (see SI Appendix, Figure S6), and the scattering intensity is primarily contributed by $\mathrm{uOmpA}_{171}$. Guinier and $\mathrm{P}(\mathrm{r})$ analyses of data collected in this condition revealed that the complex has an $R_{\mathrm{G}}$ value of $45 \pm 3 \AA$ and a $D_{\mathrm{Max}}$ of $150 \pm 10 \AA$ (Figures $2 \mathrm{~B}$ and $\mathrm{C}$, see SI Appendix, Tables S3 and 4). These sizes far exceed the expected $R_{\mathrm{G}}$ and $D_{\mathrm{Max}}$ calculated from the structure of apo-SurA $\left(R_{\mathrm{G}}=35 \AA ; D_{\mathrm{Max}}=105 \AA\right)$. Previous experiments also show that apo-SurA is 
not denatured by $\mathrm{D}_{2} \mathrm{O}(\mathrm{xx})$. We therefore conclude that the large complex size observed arises from an expanded state of $u \mathrm{OmpA}_{171}$ whilst in complex SurA.

To understand the extent to which SurA expands $\mathrm{uOmpA}_{171}$ relative to the inherent, unbound $\mathrm{uOmpA}_{171}$ molten-globule state, we estimated the intrinsic $R_{\mathrm{G}}$ and $D_{\mathrm{Max}}$ of unfolded $\mathrm{OmpA}_{171}$. It is impossible to directly measure these parameters with scattering experiments because UOMPs aggregate at the required protein concentrations. However a complementary hydrodynamic parameter of $\mathrm{uOmpA}_{171}$, the sedimentation coefficient, $s$, has been previously reported ( $s=1.65$ Svedbergs) (62). We connected $R_{G}, D_{M a x}$, and $s$-value using HullRad analysis of atomic models of $\mathrm{uOmpA}_{171}$ (63). A series of structural models of unfolded $\mathrm{OmpA}_{171}$ were

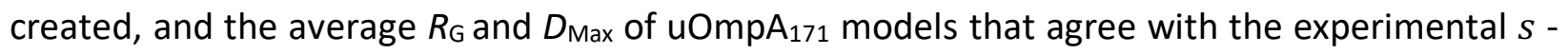
value were $25 \pm 1 \AA$ and $82.5 \pm 9 \AA$ respectively (see SI Appendix, Figures S7 and S8; error is

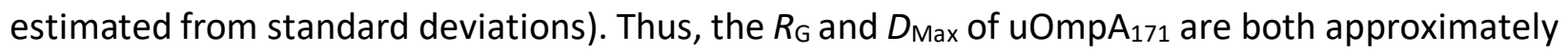
doubled when it is in complex with SurA. In sum, our pAF crosslinking and SANS data support a model where client uOMPs are expanded by SurA: a portion of the client UOMP is bound within the SurA groove, and the remainder of the uOMP is poised to sample transient interactions broadly across the SurA surface.

SurA preferentially interacts with specific segments on client uOMPs. The expansion of UOMPs by SurA raises the question of where and how they interact with the groove. To further define the molecular basis of the SurA•uOMP interaction, we used photo-crosslinking mass spectrometry (pXL-MS) to identify the segments on uOMPs that crosslinked to the high efficiency SurA $_{p A F}$ variants. We identified multiple SurA-binding segments on each client uOMP according to the following criteria.

The eight high-efficiency SurA $\mathrm{A}_{\mathrm{pAF}}$ variants were crosslinked to $\mathrm{UOmpA}_{171}$ and $\mathrm{uOmpX}$, and subjected to proteolysis with either trypsin only or trypsin and GluC in serial (see Supplementary Appendix). The resulting peptides were analyzed by LC-MS/MS, and crosslinked peptides were identified (with FDR < 0.01) using the MeroX v2.0 software package (see Supplementary Appendix) (64). Summary data of all pXL-MS experiments are given in Supplementary Appendix Table S5 and summary data of all peptide spectrum matches (PSMs) from these pXL-MS experiments are provided as Supplementary Data 1 and 2. 
Figure $3 \mathrm{~A}$ shows the crosslinking pattern identified for both $\mathrm{uOmpA}_{171}$ and $\mathrm{uOmpX}$ for each high efficiency SurA $A_{p A F}$ variant. We hypothesized that uOMP residues that repeatedly crosslink to SurA $A_{p A F}$ variants ( $>50 \%$ ) delineate preferred uOMP binding segments. This strategy was necessary because the high reactivity of the nitrene group formed upon photolysis of $p A F$ combined with the difficulty of determining the relative abundance of various crosslinked peptides made it impossible to use the crosslink sites from any individual SurA $A_{p A F}$ variant to distinguish preferred binding segments.

Using this criterion, we identified six SurA-binding segments on $\mathrm{uOmpA}_{171}$ (residues 1-21, 51-61, 84-86, 95-104, 112-113, and 130-131) and four SurA-binding segments on uOmpX (residues 29-36, 42-48, 64-73, and 81-89) as shown in Figure 3A. The identified uOMP binding segments vary in length and location between the client UOMPs and crosslink to residues on both the core and P1 domains of SurA. The sequences of these SurA-binding segments are unusually enriched in tyrosine residues (10 of the 13 tyrosines on $\mathrm{uOmpA}_{171}$ appear in segments; $\mathrm{P}=0.003$ by Chi-square test). Indeed SurA has been shown to preferentially bind to peptides with high aromatic content, affirming our criteria for defining uOMP segments (30). Serendipitously, in OmpA many of these tyrosines are highly conserved according to the Pfam database (Pfam ID: 01389), perhaps indicating importance for these residues in OmpA biogenesis (65).

To validate the importance of the SurA groove as the UOMP binding site, we performed two controls. In the first, we carried out pXL-MS on SurA $A_{422, p A F}$, which places the pAF away from the groove on the opposite side of the core domain. SurA $422, p \mathrm{AF}$ only crosslinks to a single site on $\mathrm{uOmpA}_{171}$ and did not crosslink to $\mathrm{uOmpX}$ at all, demonstrating a preference of uOMP interactions with the SurA groove (Figure 3A). Secondly, we found a similarly small number of crosslinks upon mutation of a highly conserved residue in the construct Sur $A_{26, p A F}$ that also resides away from the groove. Taken together, these experiments show that binding segments on uOMPs selectively distinguish and interact with the SurA groove.

Figure $3 \mathrm{~B}$ shows a structural model of $\mathrm{uOmpA}_{171}$ in an expanded state with a $D_{\text {Max }}$ equal to the experimentally determined size of $\mathrm{uOmpA}_{171}$ when bound to SurA. Each of the putative binding segments are highlighted with the same colors used in Figure 3A. Two possible SurA•uOmpA $\mathrm{A}_{171}$ complexes are shown with SurA bound to the second (left) and last two 
segments (right) of $u O m p A_{171}$. The presence of multiple SurA-binding segments on $u O m p A_{171}$, along with its expanded size, could allow for more than one copy of SurA to simultaneously bind to different segments of a single $u \mathrm{OmpA}_{171}$ (Figure $3 \mathrm{~B}$ ), as further supported by the presence of higher molecular weight bands in SDS-PAGE of some crosslinked SurApAF variants (see SI Appendix, Figure S2).

Modeling the Structural Features of SurA•uOmpA ${ }_{171}$. Our SDS-PAGE (Figure 1B \& SI Appendix Figure S2), SANS (Figure 2), and pXL-MS (Figure 3) experiments support a mechanism in which SurA binds a defined client UOMP segment in the SurA groove. The remainder of the UOMP is greatly expanded and presumed to be dynamic. To visualize this binding mode in more detail, we built 23 models of $\mathrm{uOmpA}_{171}$ bound to SurA using our experimental findings as restraints.

Figures S9 and S10 (see SI Appendix) provide flowcharts describing the process by which these models were generated and where each piece of experimental information was included; computational modeling is described further in SI Appendix. In essence, we docked the binding segments of $\mathrm{UOmpA}_{171}$ identified by $\mathrm{pXL-MS}$ to the groove of SurA using distance restraints generated by HADDOCK (66). The $\mathrm{uOmpA}_{171}$ component of these models were then expanded to be compatible with the SANS data (see SI Appendix, Figures S11A and B).

The Hill coefficient reported for SurA binding $\mathrm{uOmpA}_{171}$ is greater than 1, indicating more than one copy of SurA can interact with $\mathrm{uOmpA}_{171}$ at a time (23). This multiplicity is also consistent with our finding of higher molecular weight bands in SurA $A_{p A F}$ SDS-PAGE experiments. Accordingly, we also created 17 models with additional SurA protomers docked to the expanded $\mathrm{uOmpA}_{171}$ (see SI Appendix, Figure S11 C-E). Hydrodynamic parameters of each of the 40 structural models created are tabulated in Table S6.

Chemical crosslinking validates features of SurA•uOmpA 171 models, and reveals distinct binding modes. To validate structural features of the SurA $\bullet \mathrm{OmpA}_{171}$ sparse ensemble, we performed XL-MS with the chemical crosslinker disuccinimidyl dibutyric urea (DSBU) on WT SurA and our client uOMPs. In total, we identified 46 unique DSBU crosslinks between SurA and uOmpA $\mathrm{A}_{171}$ and 17 unique DSBU crosslinks between SurA and uOmpX (see SI Appendix, Figure S12, Table S5, and Supplementary Data 3-5). 
To ascertain underlying similarities in our structural models, we performed spectral biclustering on a matrix of all solvent-accessible surface distances (SASDs, calculated using JWalk) for the 46 identified crosslinks in all 23 structural models of SurA •uOmpA 171 (see SI Appendix, Figure S13 shows this analysis, Supplementary Data 6 provides all SASDs and their associated scores) (67-69). Due to the conformational heterogeneity and multiplicity of the SurA $\bullet \mathrm{uOmpA}_{171}$ sparse ensemble, no single structural model captured all of the DSBU crosslinks. However, three distinct SurA $\bullet \mathrm{UOmpA}_{171}$ binding modes emerged from this analysis, which are each explained by a unique subset of the identified crosslinks.

Figure 4A shows an example of the first binding mode, which is found in seven of our SurA $\bullet \mathrm{OOmpA}_{171}$ models, wherein segment 1 is bound in the groove and the remainder of $\mathrm{uOmpA}_{171}$ is projecting away from SurA. The second binding mode, shown in Figure 4B, is present in five SurA $\bullet \mathrm{UOmpA}_{171}$ models and is similar to binding mode one, except segment 2 is bound in the groove. Seven members of the sparse ensemble evinced a more complex topology in which the uOMP threads through the groove several times, defining a third binding mode (Figure 4C). Similar to the first binding mode, segment 1 is bound in the groove, but in this latter mode segments 3, 4, and 5 now make extensive contacts with the groove and P1 on a "second pass." Together, these three binding modes of the SurA $\bullet$ uOmpA 171 complex cover $75 \%$ (34 out of 46; see SI Appendix, Figure S13) of the identified DSBU crosslinks and help explain why so many regions of $\mathrm{UOmpA}_{171}$ can crosslink with the SurA groove (Figure 3).

As described above, we constructed $(\mathrm{SurA})_{n} \bullet \mathrm{uOmpA}_{171}$ models with higher-order stoichiometries (where $n=\{2,3,4\}$ ). These models provide an additional explanation for the complex DSBU crosslinking pattern between $\mathrm{UOmpA}_{171}$ and SurA. Figure 4D shows how a single $\mathrm{uOmpA}_{171}$ can distribute these binding modes across more than one copy of SurA instead of threading itself through a single copy of SurA multiple times - accommodating the same clusters of crosslinks. Moreover, inclusion of models with higher order stoichiometries increases coverage to $89 \%$ of identified DSBU crosslinks (Supplementary Data 6).

Finally, as a critical control to confirm the SurA groove as the primary binding site for uOMP substrates, we performed DSBU crosslinking experiments between uOmpA $\mathrm{A}_{171}$ and the “locked-closed" SurA variant (P61C/A218C) previously described by Silhavy and co-workers (38). 
In this variant the open conformation of SurA is inaccessible, and cells show increased sensitivity to viability envelope stressors in vivo. We observed a drastic reduction in the number of "lockedclosed" SurA-uOmpA 171 interprotein crosslinks compared to WT SurA (7 vs 46; see SI Appendix, Figure S12, Supplementary Data $3 \& 5$ ). This finding implies that the dense crosslinking patterns revealed by XL-MS depend on the formation of the SurA groove, and not from contacts made outside of the groove.

SANS validates and quantifies XL-MS based binding modes. To test which SurA $\bullet \mathrm{UOmpA}_{171}$ models are representative of the conformational ensemble in solution, we compared them to an

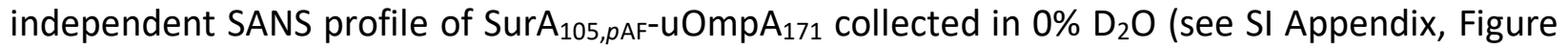
S14). In this particular condition, protonated SurA and perdeuterated $\mathrm{UOmpA}_{171}$ contribute equally to scattering (see SI Appendix, Figure S6). We calculated the expected scattering profile that would arise under these experimental conditions for each of the 40 SurA $\bullet u O m p A_{171}$ models using the software package, SASSIE (70). In addition to the models of the SurA•uOmpA $A_{171}$ complex, we included multiple models of apo-SurA in varying conformations as we were unable to completely purify the crosslinked complex (see SI Appendix, Figure S15). In agreement with the DSBU crosslinking, we found that no single model, or pairs of models, recapitulated the experimental scattering profile (reduced chi-square < 1.05) (71).

Linear combinations of the scattering profiles from three structural models were able to describe the SANS dataset. In total, we sampled over 1 million combinations of triplets of models and found 35 combinations whose simulated SANS profiles produced reduced chi-square values less than 1.05 with respect to the experimentally observed $0 \% \mathrm{D}_{2} \mathrm{O}$ SANS profile. Each accepted triplet contained at least one model of non-crosslinked SurA and one SurA $\bullet \mathrm{uOmpA}_{171}$ complex (see SI Appendix, Table S7). The three models most likely to be included in an accepted combination arise from the three distinct binding modes defined by XL-MS, and are shown in Figure 4A-C with their associated crosslinks. In addition, the linear combinations identify several models with higher-order stoichiometries as depicted in Figure 4D.

The sparse ensemble illustrating the main structural features identified by our experiments is depicted in Figure 5 by an overlay of SurA $\bullet \mathrm{uOmpA}_{171}$ models (population of each model in the ensemble are listed in Supplementary Appendix, Table S8). We note that this 
collection of conformations captures all known experimental data on SurA•uOMP complexes: including UOMP binding to the SurA groove (Figure 1), the Hill coefficient that is slightly greater than 1, the expansion of UOMPs by SurA (Figure 2), pXL-MS identification of specific binding segments (Figure 3), and the independent validation of binding modes by SANS and XL-MS (Figure 4 and 5$)(22,37,38,49)$. This sparse ensemble of models of the SurA $\bullet$ uOmpA $A_{171}$ complex provides a chemically reasonable and minimalist set of structures that could exist in the full conformational ensemble of the complex in solution. Due to limitations in the resolution of the data, additional conformations that fit the data likely exist.

\section{Discussion}

The periplasmic chaperone network is integral for $E$. coli outer membrane protein biogenesis. SurA plays several important roles in the uOMP biogenesis pathway: it must (i) recognize uOMP clients before they aggregate; (ii) maintain them in a soluble form in the periplasm; and (iii) mediate a hand-off to the BAM complex. In this study we have utilized an integrative/hybrid structural biology approach that combines multiple crosslinking and scattering techniques to generate restraints used to build a representative ensemble. This ensemble captures key structural features of SurA in complex with its client uOmpA 171 .

The model-independent Guinier analysis of the contrast-matched SANS experiments reveal that SurA performs its functions by dramatically expanding the SurA-uOMP complex in a mechanism reminiscent of trigger factor, a structural homolog to SurA (72). We observe a primary uOMP interaction in the SurA groove, located between the core and P1 domains which recapitulates recently published findings (37). The remainder of the unfolded $u O m p A_{171}$ chain that is not occupying the groove must assume an elongated conformation to be consistent with these SANS data. In contrast, a covalently "locked-closed" variant of SurA is unable to efficiently interact with $\mathrm{uOmpA}_{171}$, consistent with the reduced functionality of this variant in vivo. All together these results suggest UOMP binding to the SurA groove results in UOMP expansion relative to its compact native state, as exemplified in Figures 3, 4, and 5.

This expansion of UOMPs is distinctive from a recently published model of the SurAuOmpX complex built from crosslinking data alone (37). In that model, a single SurA completely encapsulates a globular uOMP, and this structural interpretation is incongruous with our 
experimental findings as the present SANS data demonstrate that the uOmpA $\mathrm{H}_{171}$ must instead be expanded. Although the reported crosslinking is consistent with our data, our approach also capitalized upon the usefulness of a hydrodynamic view of highly dynamic structures. Instead, our data for the SurA-uOmpA 171 complex are more consistent with a dynamic conformational ensemble proposed for the SurA-FhuA complex based on NMR (42). The extent of expansion of unfolded FhuA is unresolved, however, because the hydrodynamic properties of this system have not yet been established.

Unfolded OMP expansion mediated by a single SurA binding event is aided by the ability of additional copies of SurA to interact with distal binding segments on the UOMP (as shown in Figures 3B and 4D). Expansion of uOMPs would help avoid steric clashes between different copies of SurA simultaneously interacting with an uOMP client. For the small, eight-stranded uOMPs investigated here, higher-order stoichiometries represent minor populations in the ensemble. It is reasonable to speculate that the length of a client uOMP could dictate the binding stoichiometry of the SurA•uOMP complex as larger clients would ostensibly contain a greater number of SurA-binding segments. In accordance with this idea, gel filtration data suggest that the stoichiometry for the SurA-FhuA complex may be closer to $2: 1$ because FhuA is a much larger outer membrane protein ( $22 \beta$-strands as compared to 8 for OmpA) $(31,42)$. Higher-order stoichiometries could also be enhanced in the crowded periplasm, where the excluded volume effect increases protein-protein interactions (73).

The initial finding of $u O m p A_{171}$ expansion was surprising, especially because the other major periplasmic chaperone Skp encapsulates uOMPs in a collapsed state reminiscent of their intrinsic, unfolded conformation $(42,60)$. Given SurA interacts with uOMPs primarily through its groove, the persistent global expansion of the remainder of the client uOMP at first glance appears puzzling. We propose a kinetic trapping mechanism wherein binding and release of UOMP segments are fast relative to the collapse of the uOMP to its intrinsic molten globule state. Indeed, kinetic partitioning is a dominant organizing feature of the uOMP biogenesis chaperone network, and the interaction between SurA and UOMP happens on a very fast time scale $(18,43,74)$. Rates of unfolded uOMP collapse are not known but may be relatively slow given the low overall hydrophobicity of transmembrane $\beta$-barrel primary sequences. Such a 
difference in the rates of UOMP intrinsic collapse versus expansion by SurA binding would provide a way to retain uOMPs in an expanded state through transient repeated associations with SurA protomers. This mechanism has the advantage of limiting the amount of SurA required to solubilize a client thereby maximizing the reservoir of free SurA in the periplasm.

Figure 6 highlights several biological implications of the features of our structural ensemble. We hypothesize that these SurA properties could explain its multifaceted roles in OMP biogenesis. Firstly, we expect that expansion of uOMPs would decrease unproductive intraprotein interactions and maintain the chain in a folding-competent, unfolded conformation (Figure 6B). In this respect, SurA performs an orthogonal role to the other chaperones in the UOMP biogenesis network, which form cages around uOMPs to decrease interprotein interactions. Expansion may also mediate the formation of transient hetero-chaperone complexes in the periplasm, where more than one chaperones are simultaneously bound to an uOMP (Figure 6C and D). As uOMPs are expected to undergo hundreds individual binding and dissociation events while in the periplasm, a SurA-mediated hand-off of expanded uOMPs between chaperones would allow for these events to occur while keeping the population of aggregation-prone, unbound UOMP low (18).

An intriguing outcome of our structural ensemble is the finding that SurA-binding segments of client uOMPs are located toward the N-terminus (Figure 3). Indeed, the three binding modes found for the SurA $\bullet \mathrm{UOmpA}_{171}$ interaction are mediated by the two most Nterminal binding segments on $\mathrm{uOmpA}_{171}$. This suggests the possibility of an early encounter between SurA and client uOMPs in the periplasm (Figure 6A). Accordingly, a recent, low resolution cryo-EM model places SurA near the translocon where it is well positioned to bind emerging uOMP segments (75).

Conversely, there was a conspicuous absence of robust crosslinking for both client OMPs near their C-termini. The apparent lack of SurA interaction sites in this UOMP region leaves the $\beta$-signal (Aro-X-Aro) free to interact with other members of the UOMP biogenesis pathway. The $\beta$-signal has been shown to play an important role in mediating efficient catalysis of UOMP folding by BAM both in vivo and in vitro (76-78). As SurA is the only periplasmic chaperone that promotes the interaction between UOMPs and BAM, our data support a 
mechanistic hypothesis in which this region of uOMPs is free and flexible and effectively 'cast' outward in a mechanism reminiscent of fly fishing to catch the BAM complex (Figure 6E) (13, 79).

Even if it is transient, the formation of a SurA $\bullet$ UOMP•BAM ternary complex is enticing because it brings the uOMP close to both the BAM complex and the disrupted adjacent membrane, both of which accelerate uOMP folding $(9,80)$. The BAM complex has been proposed to template and insert UOMP $\beta$-hairpins as individual foldamers of OMPs. Notably, the SurA groove is large enough to accommodate a $\beta$-hairpin, which could potentially favor the pre-formation of this key structural elements in a nascent uOMP. Moreover, this SurAmediated $\beta$-hairpin formation mechanism could be easily adapted to larger clients with more transmembrane strands (and probably more SurA-binding segments) given the modular nature of the $\beta$-hairpin unit.

In this work we highlight the utility and complementarity of photo- and chemical crosslinking, neutron scattering, and mass spectrometry applied together. This combined approach was crucial because unfolded proteins present many challenges to conventional structural techniques due of their absence of regular secondary structure, their high conformational flexibility, and their propensity to aggregate. Our results illuminate a sparse ensemble of models that captures the key structural features defining how SurA promotes outer membrane protein biogenesis. 


\section{Materials and Methods}

SurA Expression and Purification- The SI Appendix describes creation, expression, and purification of all SurA constructs used in this study.

pAF Crosslinking- $25 \mu$ mols $\mathrm{L}^{-1}(\mu \mathrm{M})$ of each SurA $\mathrm{A}_{p \mathrm{~F}}$ variant was mixed with $5 \mu \mathrm{M}$ uOMP (20 mmoles $\mathrm{L}^{-1}(\mathrm{mM})$ Tris, $1 \mathrm{~mol} \mathrm{~L}^{-1}(\mathrm{M})$ urea, $\mathrm{pH}=$ 8.0. uOMPs expressed and purified as described previously (81). We chose these conditions because both SurA and $u \mathrm{OmpA}_{171}$ are monomeric and soluble at the listed protein and urea concentrations $(39,51)$. Mixtures were then irradiated with UV light (wavelength, $\lambda=254 \mathrm{~nm}$ ) for 5 minutes using a Spectroline MiniMax UV Lamp (Fisher \#11-992-662). Aliquots were taken for SDS-PAGE analysis both pre- and postexposure to UV light. These samples were subjected to electrophoresis using a 4-20\% gradient precast gel (Mini-PROTEAN TGX, Bio-Rad) at a constant voltage of $200 \mathrm{~V}$ for 35 minutes at room-temperature.

Using ImageJ, densitometry analysis on the loss of density of the $\mathrm{uOmpA}_{171}$ band was utilized to quantitate crosslinking efficiency. Crosslinking efficiency values were corrected for the amount of $\mathrm{OOmpA}_{171}$ band lost ( $20 \%$ ) when mixed with WT SurA (not containing $p A F$ ). A representative SDS-PAGE gel for each SurA $A_{p A F}$ variant and $\mathrm{uOmpA}_{171}$ is shown in the $\mathrm{SI}$ Appendix, Figure S2. This same protocol was utilized to assess the crosslinking of SurA $A_{p A F}$ variants to $\mathrm{uOmpX}$ and $\mathrm{uOmpLA}$.

SANS on Protonated-SurA/Perdeuterated-uOmpA ${ }_{171}$ Complex- SurA $_{105, p A F}$ was crosslinked to deuterated-uOmpA 171 as described above. Perdeuterated $\mathrm{OmpA}_{171}$ growth, expression, purification, and characterization are detailed in the SI Appendix. This complex was further purified via size exclusion chromatography (GE Superdex-200 10/300 GL; flowrate = 0.6 $\mathrm{mL} / \mathrm{min}$ ) in $20 \mathrm{mM}$ Tris, $200 \mathrm{mM} \mathrm{NaCl}, \mathrm{pH} 8.0$ (GF buffer), and buffer exchanged into either $0 \%$ or $30 \% \mathrm{D}_{2} \mathrm{O}$ for SANS experiments (same buffer components as SEC) (see SI Appendix, Figure S15). We made three attempts to also collect scattering profiles in $80 \%$ and $98 \% \mathrm{D}_{2} \mathrm{O}$ of this complex but the I(0) values from Guinier fitting indicated that these samples contained aggregates. It is known that increased buffer concentrations of $\mathrm{D}_{2} \mathrm{O}$ may promote selfassociation and aggregation of particularly hydrophobic proteins (82). 
All scattering experiments were collected at the National Institute of Standards and Technology Center for Neutron Research (Gaithersburg, MD) as previously described(60). More information on SANS data collection and analysis are found in the SI Appendix.

XL-MS of SurA-uOMP complexes - The SI Appendix describes the methods used to perform

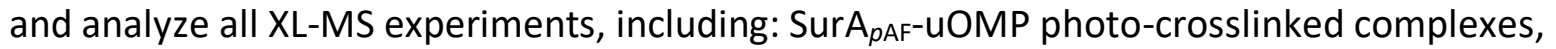
SurA-uOMP DSBU crosslinked complexes, and "locked closed" SurA-uOmpA 171 DSBU crosslinking complexes. This includes pAF and DSBU methods and data analysis protocols.

Structural models of SurA - Models of SurA were constructed based on crystal structures 1M5Y and 2PV3 (Table S2). In the 1M5Y structure, the core and P1 domains are close together but the P2 domain is extended. In the 2PV3 structure, the P1 domain is moved away from the core domain and rotated relative to $1 \mathrm{M} 5 \mathrm{Y}$ but the P2 domain is missing. Residue segments $20-34,165-$ $171,387-394$, and $428-430$ were built into $1 \mathrm{M} 5 \mathrm{Y}$ and six histidine residues were added to the Cterminus using Modeler to create the "P1 closed" form of SurA (83). For the "P2 closed" form of SurA, PyMOL was used to build the P2 domain from the P1 closed form into 2PV3 (84). The P2 domain was then moved into position against the groove formed by the core and P1 domains using NAMD as described below. The "open" SurA model has the core-P1 relative orientation from $2 \mathrm{PV} 3$ and the core-P2 relative orientation from 1M5Y. Domains were oriented in PyMOL and linkage conformations were normalized using NAMD as described below. The "Collapsed" SurA model has the core-P1 relative orientation from $1 \mathrm{M} 5 \mathrm{Y}$ and the $\mathrm{P} 2$ domain was moved into position against the core using NAMD as described below.

The P2 closed, open, and collapsed SurA models, initially constructed using PyMOL, were further manipulated to position the domains, remove Van der Waals clash and relax unstructured linkage segments using NAMD with generalized Born implicit solvent electrostatics in the CHARMM22 force field (85). Domains were positioned with targeted distance restraints as implemented in the collective variable module in $\operatorname{NAMD}(86,87)$. Typically, a harmonic potential was placed on the distance between the centers of mass of two groups of CA atoms with a force constant of $1.0 \mathrm{kcal} / \mathrm{mol}$ and the force was applied for 50,000 to 150,000 steps. This in vacuo molecular relaxation and manipulation was carried out after 200 steps of energy minimization, with implicit solvent alpha cutoff $=12.0 \AA$, [ion] $=0.3 \mathrm{M}$, non- 
bonded cutoff $=14.0$, switching starting at 13.0, and 2 fs time step. Langevin dynamics was used with a damping coefficient of 1 for temperature control (NVT). The domain-domain distances of SurA were monitored during simulation and a structure was saved when target distances were obtained.

Structural models of the SurA•uOmpA $A_{171}$ complex. Four extended $\mathrm{uOmpA}_{171}$ segments (residues 2-21, 54-73, 84-104, 112-132) that contain the six SurA-binding segments were independently submitted, along with the open SurA structural model to the protein-protein docking web server HADDOCK (66). These sequence segments were chosen to include those residues that were found to repeatedly crosslink to the high efficiency SurA $A_{p A F}$ variants. Active and passive residues for HADDOCK were chosen from SurA groove (see SI Appendix, Figure S9).

These docked oligopeptides were inspected using molecular graphics to obtain target

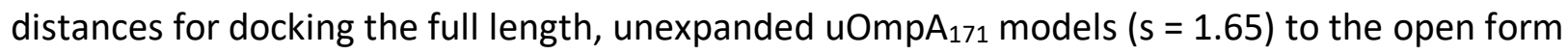
of SurA. Docking was accomplished in NAMD using the target distances from HADDOCK peptide docking as distance restraints in the collective variables module of NAMD as described above.

The uOmpA-open SurA models were further manipulated to increase the $u O m p A D_{\operatorname{Max}}$ to the target of $150 \AA$ that was determined by $P(r)$ analysis of the SANS data. These expansions were accomplished using distance restraints and the collective variable module in NAMD. Short segments of each bound uOmpA that were furthest apart were identified and the two groups of respective CA atoms were forced to a distance of $\sim 150 \AA ̊$ with a harmonic potential as described above. A second open SurA model was then docked to exposed, known binding segments (see SI Appendix, Figure S11) of the extended uOmpA. In three cases, a third open SurA was docked to remaining exposed known binding segments. One extended polypeptide of uOmpA was generated with a $D_{\operatorname{Max}} \sim 250 \AA$ and four open SurA models were docked to the four main segments on OmpA that displayed high efficiency cross-linking.

In all, twenty-three models containing one docked SurA, thirteen models containing two docked SurA, three models containing three SurA, and one model containing four SurA were built. Physical dimensions of these models are listed in Table S6. Values for $R_{\mathrm{G}}$ and $D_{\mathrm{Max}}$ were calculated using HullRad(63). All models contained CHARMM hydrogens and were used to calculate predicted SANS profiles using the SasCalc server.(70) 
Methods and information regarding the comparison of structural models to the $0 \% \mathrm{D}_{2} \mathrm{O}$ SANS profiles performed to generate the ensemble of structures shown in Figure 5 are found in detail in the SI Appendix. Models included in the sparse conformational ensemble of SurA-

uOmpA171 can be found at: https://github.com/KarenGFleming/SurAuOmpA. 


\section{Acknowledgements}

We thank the Johns Hopkins University Biomolecular NMR Center and the Center for Molecular Biophysics for providing facilities and resources. Access to NGB30 SANS was provided by the Center for High Resolution Neutron Scattering, a partnership between the National Institute of Standards and Technology and the National Science Foundation under Agreement No. DMR1508249. We acknowledge the support of the National Institute of Standards and Technology, U.S. Department of Commerce, in providing the neutron research facilities used in this work. Certain commercial equipment, instruments, or materials (or suppliers, or software, ...) are identified in this paper to foster understanding. Such identification does not imply recommendation or endorsement by the National Institute of Standards and Technology, nor does it imply that the materials or equipment identified are necessarily the best available for the purpose. This work benefitted from CCP-SAS software developed through a joint EPSRC (EP/K039121/1) and NSF (CHE-1265821) grant. This work was supported by National Science Foundation (NSF) MCB1412108 and NIH R01 GM079440 grants (to K.G.F.). D.C.M, A.M.P., T.D. and H.J.L. were supported by NIH training grant T32 GM008403. A.M.P. was supported by the NSF grant DGE 1232825. The authors thank lab members for helpful discussions. 

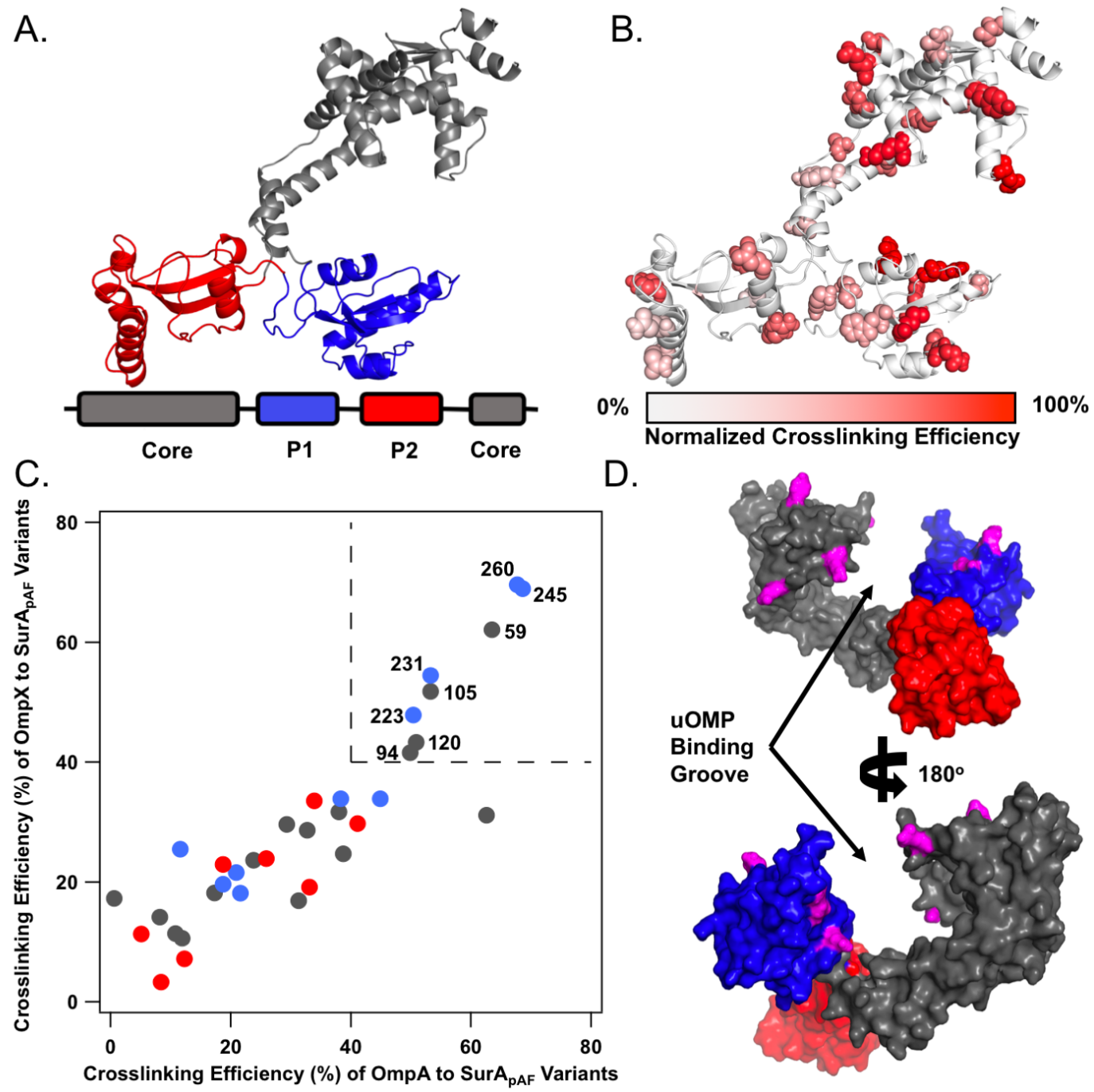

D.

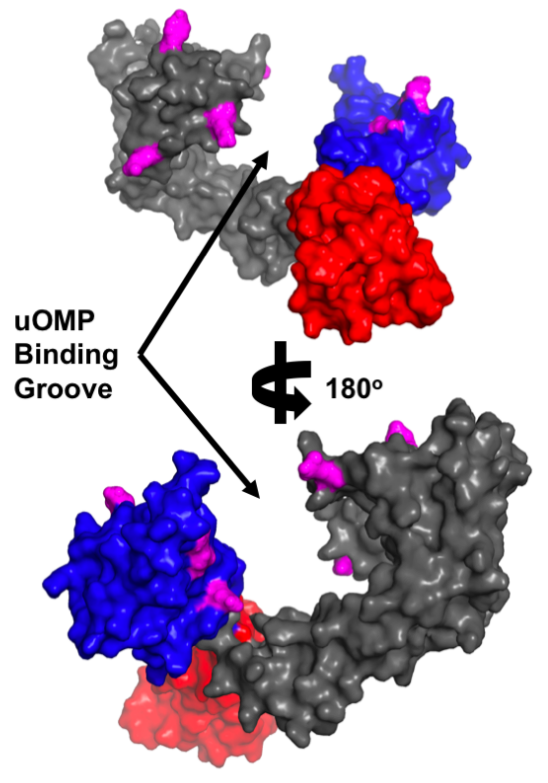

Figure 1. Open SurA binds client uOMPs in a groove between domains. (A) The structure of open SurA shown as a cartoon, with the domains colored as depicted in the sequence diagram below (core: gray, P1: blue, P2: red). In this conformation, the three domains of SurA are structurally isolated from each other and do not form extensive inter-domain contacts. (B) The 32 surface exposed sites on SurA in which pAF was substituted, shown in a space-filling representation. Photo-crosslinking was induced with 5-min UV exposure. Each crosslinking site is colored based on the normalized crosslinking efficiency to $\mathrm{UOmpA}_{171}$ as based on quantitative SDS-PAGE (see SI Appendix, Figure S2). The highest crosslinking sites are found on the core and P1 domains of SurA, while P2 exhibits only modest crosslinking efficiency. (C) The raw crosslinking efficiencies of SurA to $\mathrm{uOmpA}_{171}$ and $\mathrm{uOmpX}$ are shown and colored by the SurA domain in which they residue (as in (A)). Eight SurA $A_{p A F}$ variants stand out by having high (>40\%) crosslinking efficiency to both uOMP clients, and are labeled with their residue number in the upper right quadrant of the graph (demarcated by dotted lines). (D) The eight high efficiency crosslinking sites, shown in magenta, are mapped on to a surface representation of the structure of open SurA. Together, these sites line a groove formed between the core and P1 domains, indicating that UOMPs are primarily bound there. 

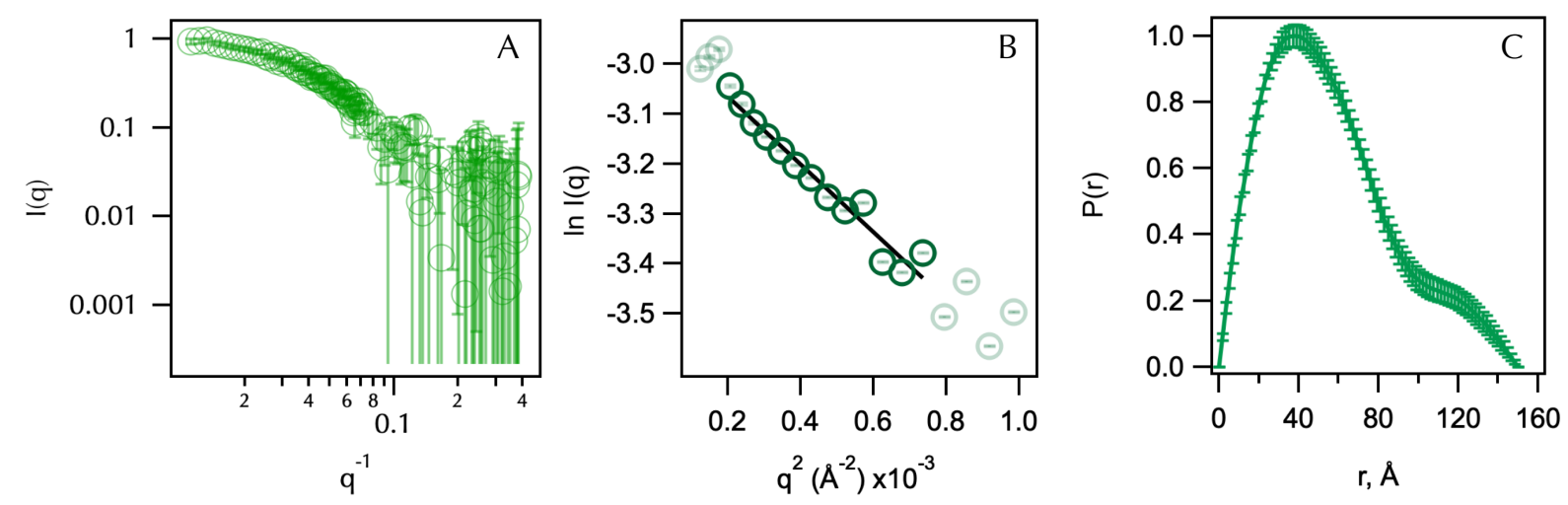

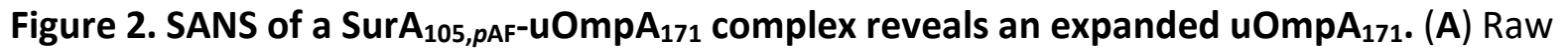
scattering profile of protonated-SurA $A_{105, p A F}$ photo-crosslinked to deuterated-uOmp $A_{171}$ in $30 \%$ $\mathrm{D}_{2} \mathrm{O}$ buffer is shown in green. Error bars represent the standard error of the mean with respect to the number of pixels used in the data averaging. (B) Linear fit of the Guinier region of the SANS profile determines the $R_{\mathrm{G}}$ of the complex to be $45 \pm 3 \AA$. (C) $P(R)$ distribution function; $D_{\text {Max }}$ is estimated to be $150 \pm 10 \AA$. 


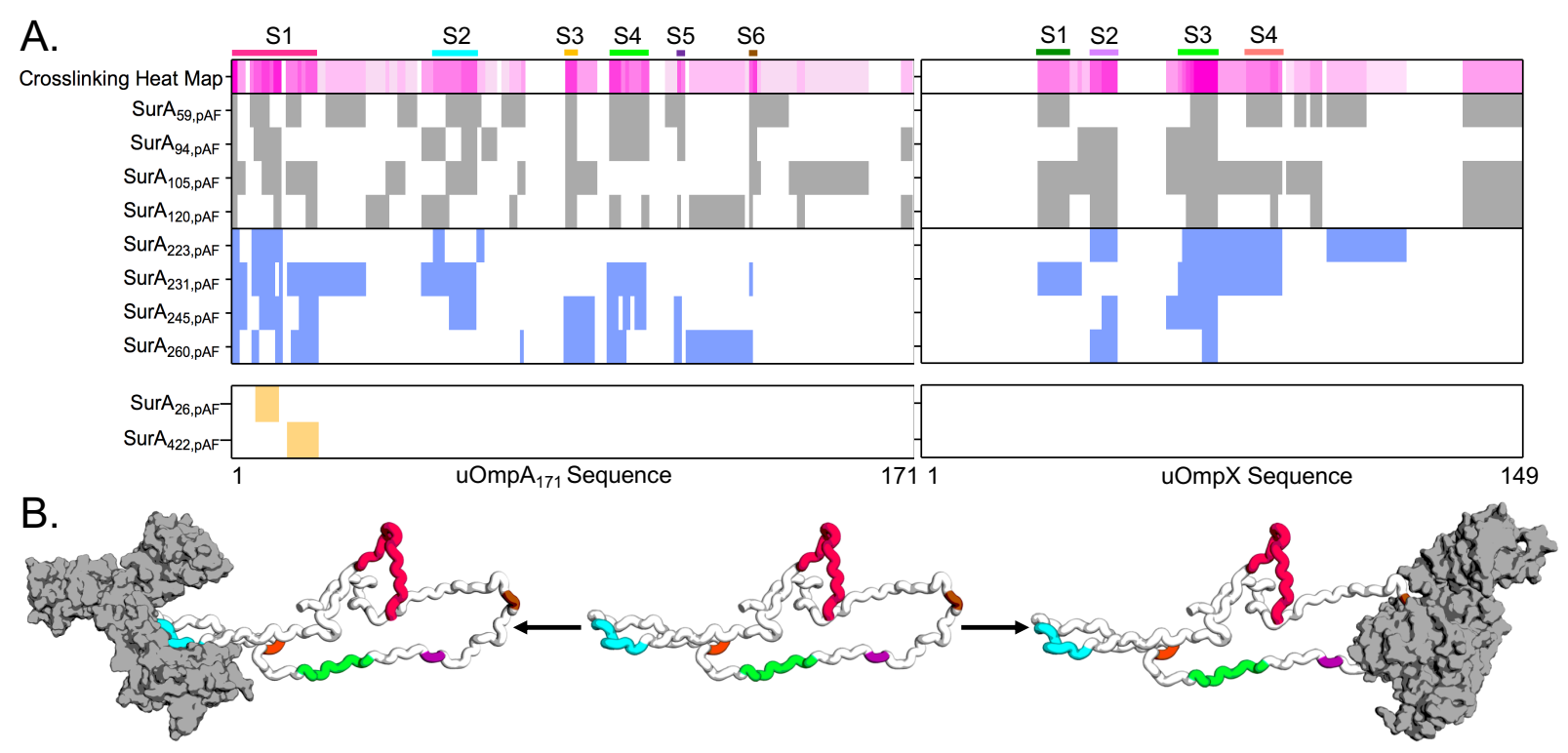

Figure 3. Photo-crosslinking mass spectrometry ( $p X L-M S)$ identifies segments on client uOMPs that bind SurA. (A) The crosslinking patterns for eight high-efficiency SurA $A_{p A F}$ variants

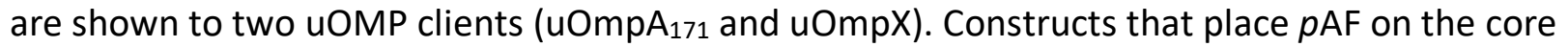
domain are colored gray (top register) and constructs that place $p A F$ on the P1 domain are colored blue (second register). The crosslinking heat map depicts the frequency a given residue on a client UOMP crosslinks to $\mathrm{pAF}$ in eight separate crosslinking experiments (darker magenta indicates residue is crosslinked more often). Binding segments are demarcated with a colored bar above the heat map and a label (S1-6 or S1-4). The bottom register shows results from two negative control studies using SurA $A_{26, p A F}$ and SurA $A_{422, p A F}$ (see main text for explanation). Only one $\mathrm{uOmpA}_{171}$ crosslinked peptide was found for each of these constructs, while uOmpX did not crosslink at all. (B) An expanded $u \mathrm{OmpA}_{171}$ model with hydrodynamic properties matching the contrast-matched SANS experiment is shown as a cartoon with the SurA-binding segments colored as in (A). Two different segments (S2, left, o1s022 and S6, right, o1s021) are shown bound to SurA (shown in gray with a surface representation), suggesting that more than one copy of SurA could bind a single copy of $\mathrm{uOmpA}_{171}$ with minimal steric clash. 


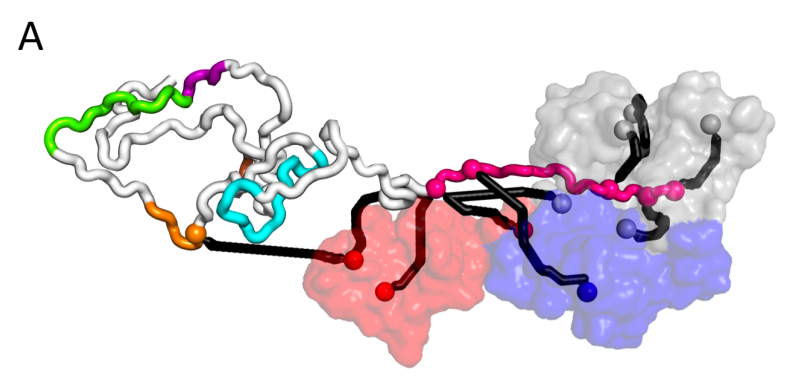

B

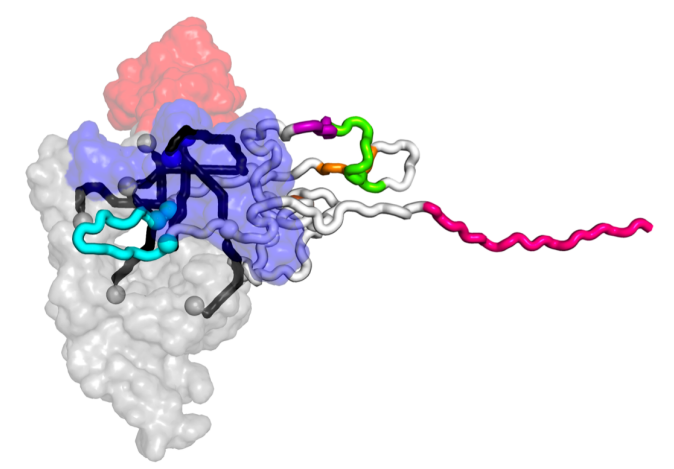

C
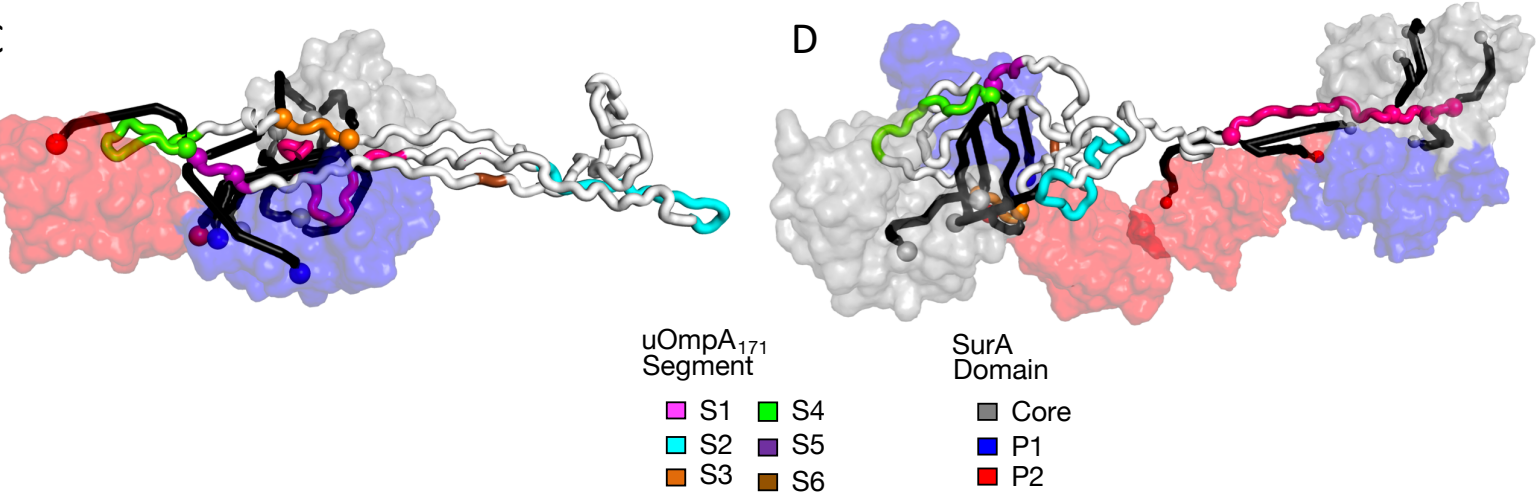

$$
\begin{aligned}
& \text { SurA } \\
& \text { Domain } \\
& \square \text { Core } \\
& \square \text { P1 } \\
& \square \text { P2 }
\end{aligned}
$$

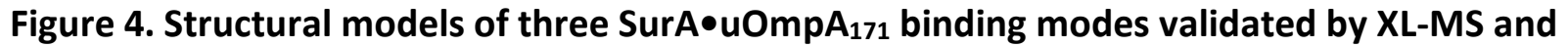
SANS. SurA is represented as a surface (colored as in Figure $1 \mathrm{~A}$ ) and $u \mathrm{OmpA}_{171}$ is represented as a tube (with binding segments demarcated by color as in Figure 3). DSBU crosslink sites are represented as spheres with crosslinks depicted as SASDs created by Jwalk. Crosslinks captured in each model are colored black (see SI Appendix, Figure S13 for details). (A) A representative structural model of the first binding mode (01s016), supported by a cluster of ten crosslinks primarily between S1 (pink) and the core and P2 domains of SurA. (B) A representative structural model of the second binding mode (01s010), supported by a cluster of nine crosslinks primarily between $\mathrm{S} 2$ (cyan) and surrounding regions of $\mathrm{uOmpA}_{171}$ and the core and $\mathrm{P} 1$ domains of SurA. (C) Representative structural model of the third binding mode (01s009), supported by fifteen crosslinks between segment 1 (pink) and segments 3, 4, and 5 (orange) primarily to the core domain of SurA. (D) A representative structure of two SurA protomers bound to $\mathrm{UOmpA}_{171}$ (o2s006), wherein one copy of SurA binds segment 1, and another copy binds segments 3,4 , and 5. 


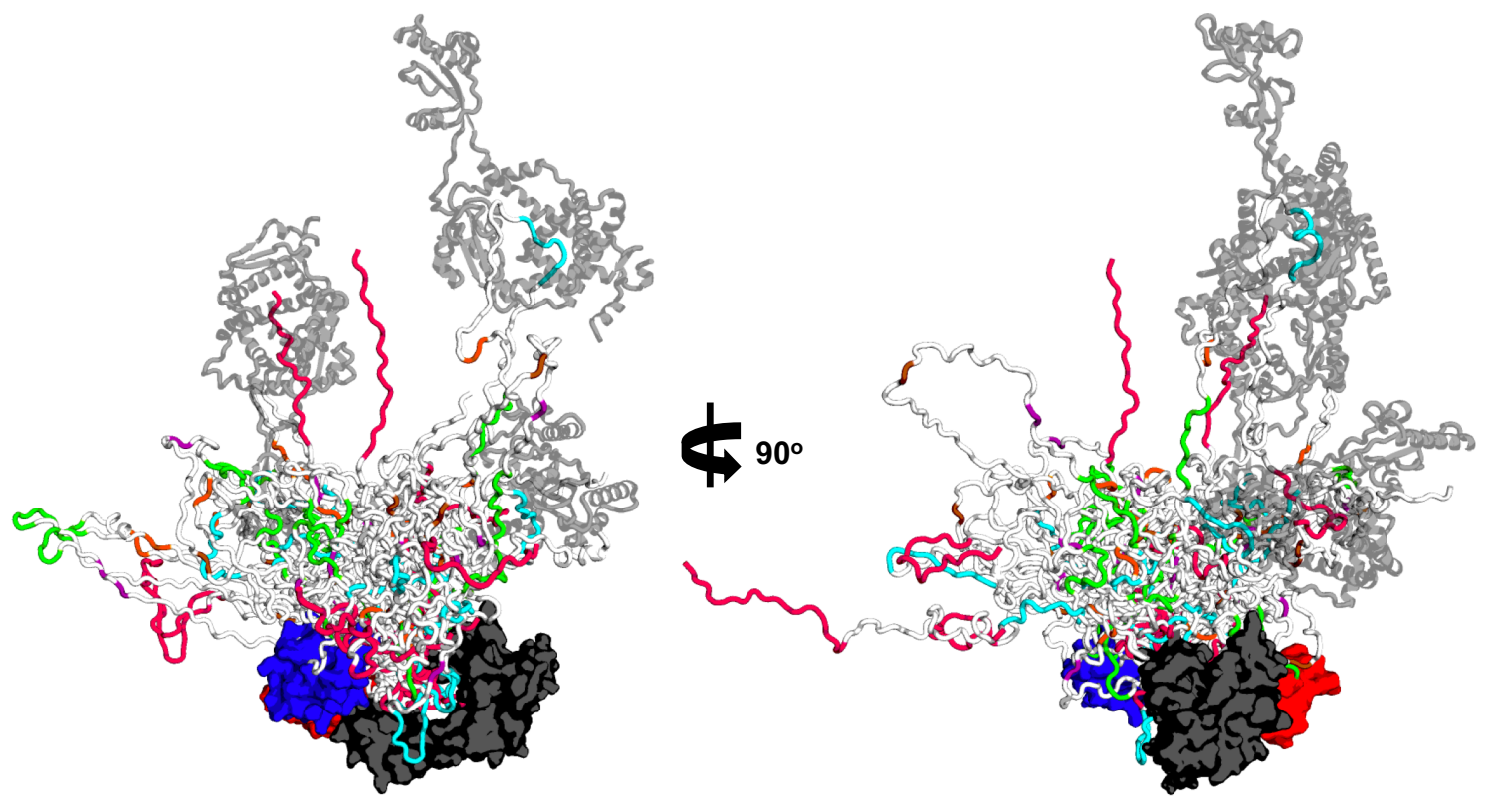

Figure 5. SurA•uOmpA $A_{171}$ ensemble as defined by experimental restraints reveals uOmpA $\mathrm{A}_{171}$ conformational landscape. The 21 SurA $\bullet \mathrm{OmpA}_{171}$ structural models that were part of triplets of linearly weighted models that fit the $0 \% \mathrm{D}_{2} \mathrm{O}$ SANS data are overlaid aligned to SurA. SurA is in the open conformation and shown with a surface representation with domains colored as in Figure $1 \mathrm{~A}$. uOmpA $\mathrm{A}_{171}$ models are shown with a cartoon representation and have SurA-binding segments, as defined by XL-MS, colored as in Figure 3. Higher order stoichiometries are found in the ensemble, with additional copies of SurA shown as transparent, gray cartoons. The diversity of $\mathrm{uOmpA}_{171}$ conformations that are shown in this ensemble highlight the conformational dynamics accessible to client uOMPs when bound to SurA. 


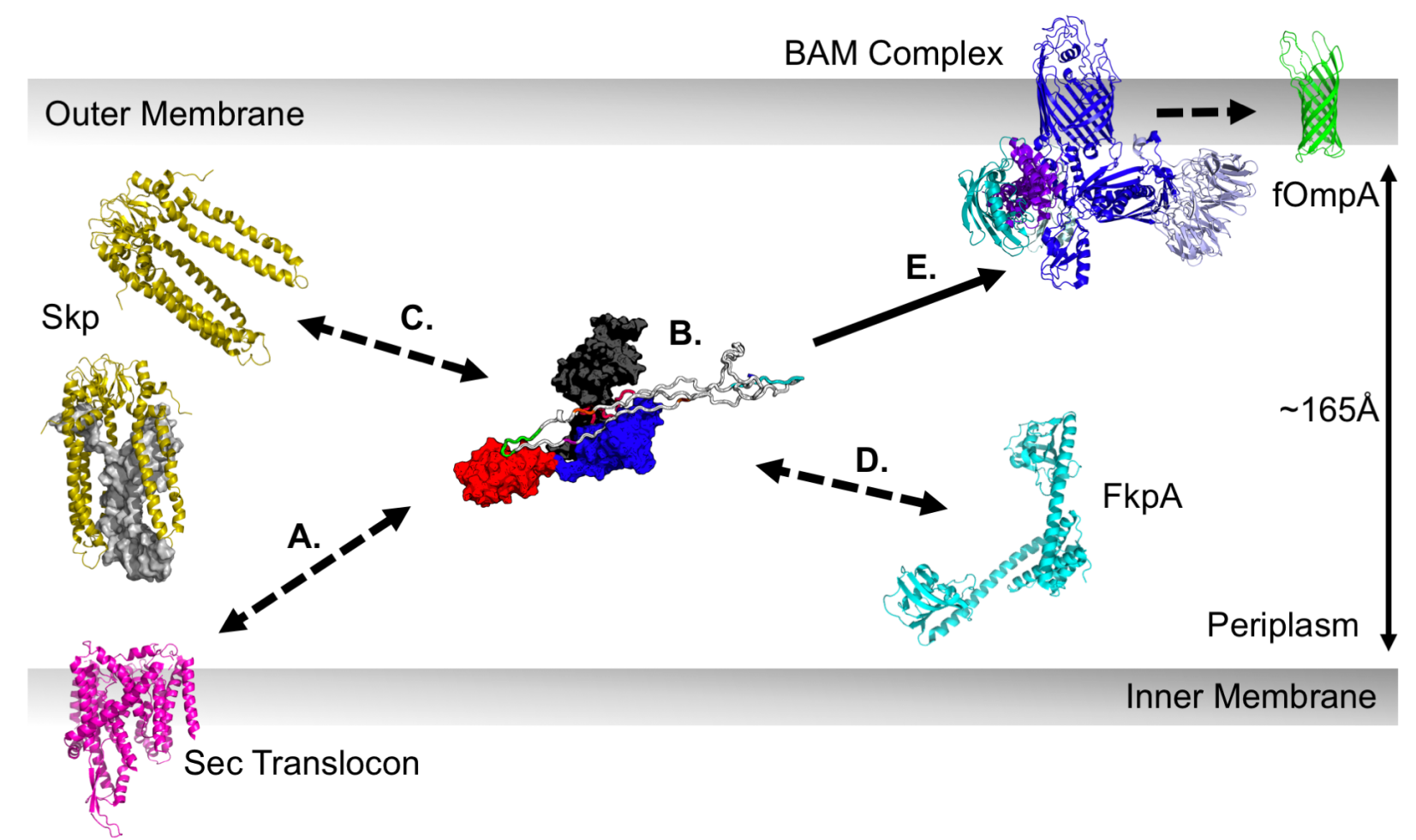

Figure 6. Implications of uOMP expansion in the periplasm. uOMPs are post-translationally secreted through the Sec translocon (magenta), N-to-C terminally. (A) The emerging uOMP Nterminus in the periplasm may be recognized by SurA. (B) After complete translocation into the periplasm, one or more SurA protomers bind specific segments on UOMP clients, solubilizing the UOMP in expanded conformations nearly the width of the periplasm. The expanded uOMP may also be able to form heterocomplexes with other chaperones in the OMP biogenesis pathway: Skp (C) and FkpA (D). The size of uOMPs bound to SurA is approximately double the size of uOMPs bound to Skp. Unfolded OmpA $\mathrm{m}_{171}$ bound to $\mathrm{Skp}$ is shown as a gray surface representation in the Skp trimer located proximal to the translocon. (E) The extended, unbound C-terminal region of the SurA-bound UOMP is positioned to encounter the BAM complex, which recognizes the OMP $\beta$-signal and catalyzes UOMP folding into the outer membrane. 


\section{References}

1. F. Chiti, C. M. Dobson, Protein Misfolding, Amyloid Formation, and Human Disease: A Summary of Progress Over the Last Decade. Annu. Rev. Biochem. 86, 27-68 (2017).

2. C. M. Dobson, Principles of protein folding, misfolding and aggregation in Seminars in Cell and Developmental Biology, (Elsevier Ltd, 2004), pp. 3-16.

3. J. M. Barral, S. A. Broadley, G. Schaffar, F. U. Hartl, Roles of molecular chaperones in protein misfolding diseases. Semin. Cell Dev. Biol. 15, 17-29 (2004).

4. F. U. Hartl, M. Hayer-Hartl, Protein folding. Molecular chaperones in the cytosol: From nascent chain to folded protein. Science (80-. ). 295, 1852-1858 (2002).

5. Y. E. Kim, M. S. Hipp, A. Bracher, M. Hayer-Hartl, F. Ulrich Hartl, Molecular Chaperone Functions in Protein Folding and Proteostasis. Annu. Rev. Biochem. 82, 323-355 (2013).

6. H. Saibil, Chaperone machines for protein folding, unfolding and disaggregation. Nat. Rev. Mol. Cell Biol. 14, 630-642 (2013).

7. F. U. Hartl, Molecular chaperones in cellular protein folding. Nature 381, 571-580 (1996).

8. F. U. Hartl, A. Bracher, M. Hayer-Hartl, Molecular chaperones in protein folding and proteostasis. Nature 475, 324-332 (2011).

9. A. M. Plummer, K. G. Fleming, From Chaperones to the Membrane with a BAM! Trends Biochem. Sci. 41, 872-882 (2016).

10. D. Chaturvedi, R. Mahalakshmi, Transmembrane $\beta$-barrels: Evolution, folding and energetics. Biochim. Biophys. acta. Biomembr. 1859, 2467-2482 (2017).

11. T. Ulrich, D. Rapaport, Biogenesis of beta-barrel proteins in evolutionary context. Int. J. Med. Microbiol. 305, 259-64 (2015).

12. J. M. Herrmann, J. Riemer, Three approaches to one problem: protein folding in the periplasm, the endoplasmic reticulum, and the intermembrane space. Antioxid. Redox Signal. 21, 438-56 (2014).

13. Y. Wang, et al., A supercomplex spanning the inner and outer membranes mediates the biogenesis of $\beta$-barrel outer membrane proteins in bacteria. J. Biol. Chem. 291, 1672016729 (2016).

14. R. Koebnik, K. P. Locher, P. Van Gelder, Structure and function of bacterial outer 
membrane proteins: Barrels in a nutshell. Mol. Microbiol. 37, 239-253 (2000).

15. A. Luther, et al., Chimeric peptidomimetic antibiotics against Gram-negative bacteria. Nature 576, 452-458 (2019).

16. E. M. Hart, et al., A small-molecule inhibitor of BamA impervious to efflux and the outer membrane permeability barrier. Proc. Natl. Acad. Sci. U. S. A. 116, 21748-21757 (2019).

17. Y. Imai, et al., A new antibiotic selectively kills Gram-negative pathogens. Nature 576, 459-464 (2019).

18. S. M. Costello, A. M. Plummer, P. J. Fleming, K. G. Fleming, Dynamic periplasmic chaperone reservoir facilitates biogenesis of outer membrane proteins. Proc. Natl. Acad. Sci. U. S. A. 113, E4794-E4800 (2016).

19. J. G. Sklar, T. Wu, D. Kahne, T. J. Silhavy, Defining the roles of the periplasmic chaperones SurA, Skp, and DegP in Escherichia coli. Genes Dev. 21, 2473-2484 (2007).

20. A. E. Rizzitello, J. R. Harper, T. J. Silhavy, Genetic evidence for parallel pathways of chaperone activity in the periplasm of Escherichia coli. J. Bacteriol. 183, 6794-6800 (2001).

21. T. J. Silhavy, J. C. Malinverni, Assembly of Outer Membrane $\beta$-Barrel Proteins: the Bam Complex. EcoSal Plus 4 (2011).

22. J. R. Humes, et al., The Role of SurA PPlase Domains in Preventing Aggregation of the Outer-Membrane Proteins tOmpA and OmpT. J. Mol. Biol. 431, 1267-1283 (2019).

23. B. Schiffrin, et al., Effects of Periplasmic Chaperones and Membrane Thickness on BamACatalyzed Outer-Membrane Protein Folding. J. Mol. Biol. 429, 3776-3792 (2017).

24. G. Mas, S. Hiller, Conformational plasticity of molecular chaperones involved in periplasmic and outer membrane protein folding. FEMS Microbiol. Lett. 365 (2018).

25. G. Mas, J. Thoma, S. Hiller, The Periplasmic Chaperones Skp and SurA. Subcell. Biochem. 92, 169-186 (2019).

26. A. P. Chum, S. R. Shoemaker, P. J. Fleming, K. G. Fleming, Plasticity and transient binding are key ingredients of the periplasmic chaperone network. Protein Sci. 28, 1340-1349 (2019).

27. F. Stull, J.-M. Betton, J. C. A. Bardwell, Periplasmic Chaperones and Prolyl Isomerases. 
EcoSal Plus 8 (2018).

28. K. Denoncin, J. Schwalm, D. Vertommen, T. J. Silhavy, J.-F. Collet, Dissecting the Escherichia coli periplasmic chaperone network using differential proteomics. Proteomics 12, 1391-401 (2012).

29. S. W. Lazar, M. Almirón, A. Tormo, R. Kolter, Role of the Escherichia coli SurA protein in stationary-phase survival. J. Bacteriol. 180, 5704-11 (1998).

30. G. Hennecke, J. Nolte, R. Volkmer-Engert, J. Schneider-Mergener, S. Behrens, The periplasmic chaperone SurA exploits two features characteristic of integral outer membrane proteins for selective substrate recognition. J. Biol. Chem. 280, 23540-23548 (2005).

31. D. Vertommen, N. Ruiz, P. Leverrier, T. J. Silhavy, J. F. Collet, Characterization of the role of the escherichia coli periplasmic chaperone SurA using differential proteomics.

Proteomics 9, 2432-2443 (2009).

32. S. E. Rollauer, M. A. Sooreshjani, N. Noinaj, S. K. Buchanan, Outer membrane protein biogenesis in Gram-negative bacteria. Philos. Trans. R. Soc. Lond. B. Biol. Sci. 370 (2015).

33. B. Schiffrin, et al., Skp is a multivalent chaperone of outer-membrane proteins. Nat. Struct. Mol. Biol. 23, 786-793 (2016).

34. T. Krojer, et al., Structural basis for the regulated protease and chaperone function of DegP. Nature 453, 885-890 (2008).

35. C. W. Sandlin, N. R. Zaccai, K. G. Fleming, Skp Trimer Formation Is Insensitive to Salts in the Physiological Range. Biochemistry 54, 7059-62 (2015).

36. E. Bitto, D. B. McKay, Crystallographic structure of SurA, a molecular chaperone that facilitates folding of outer membrane porins. Structure 10, 1489-98 (2002).

37. A. N. Calabrese, et al., Inter-domain dynamics in the chaperone SurA and multi-site binding to its outer membrane protein clients. Nat. Commun. 11, 1-16 (2020).

38. G. R. Soltes, J. Schwalm, D. P. Ricci, T. J. Silhavy, The activity of Escherichia coli chaperone SurA is regulated by conformational changes involving a parvulin domain. J. Bacteriol. 198, 921-929 (2016).

39. D. C. Marx, M. J. Leblanc, A. M. Plummer, S. Krueger, K. G. Fleming, Domain Interactions 
Determine the Conformational Ensemble of the Periplasmic Chaperone SurA. Protein Sci. (2020) https:/doi.org/10.1002/pro.3924.

40. H. M. Webb, L. W. Ruddock, R. J. Marchant, K. Jonas, P. Klappa, Interaction of the periplasmic peptidylprolyl cis-trans isomerase SurA with model peptides: The N-terminal region of SurA is essential and sufficient for peptide binding. J. Biol. Chem. 276, 4562245627 (2001).

41. S. Behrens, R. Maier, H. de Cock, F. X. Schmid, C. A. Gross, The SurA periplasmic PPlase lacking its parvulin domains functions in vivo and has chaperone activity. EMBO J. 20, 285-94 (2001).

42. J. Thoma, B. M. Burmann, S. Hiller, D. J. Müller, Impact of holdase chaperones Skp and SurA on the folding of $\beta$-barrel outer-membrane proteins. Nat. Struct. Mol. Biol. advance on, $795-802$ (2015).

43. G. Li, et al., Single-Molecule Detection Reveals Different Roles of Skp and SurA as Chaperones. ACS Chem. Biol. 13, 1082-1089 (2018).

44. E. W. Bell, E. J. Zheng, L. M. Ryno, Identification of inhibitors of the E. coli chaperone SurA using in silico and in vitro techniques. Bioorg. Med. Chem. Lett. 28, 3540-3548 (2018).

45. E. Bitto, D. B. McKay, Binding of phage-display-selected peptides to the periplasmic chaperone protein SurA mimics binding of unfolded outer membrane proteins. FEBS Lett. 568, 94-98 (2004).

46. K. H. Stymest, P. Klappa, The periplasmic peptidyl prolyl cis-trans isomerases PpiD and SurA have partially overlapping substrate specificities. FEBS J. 275, 3470-3479 (2008).

47. E. Bitto, D. B. McKay, The periplasmic molecular chaperone protein SurA binds a peptide motif that is characteristic of integral outer membrane proteins. J. Biol. Chem. 278, 49316-49322 (2003).

48. Q. Chai, et al., Diverse sequences are functional at the C-terminus of the E. coli periplasmic chaperone SurA. Protein Eng. Des. Sel. 27, 111-116 (2014).

49. J. Thoma, B. M. Burmann, S. Hiller, D. J. Müller, Impact of holdase chaperones Skp and SurA on the folding of $\beta$-barrel outer-membrane proteins. Nat. Struct. Mol. Biol. 22, 795802 (2015). 
50. G. Krainer, et al., Slow Interconversion in a Heterogeneous Unfolded-State Ensemble of Outer-Membrane Phospholipase A (2017) https:/doi.org/10.1016/j.bpj.2017.05.037 (March 6, 2020).

51. A. E. Tan, N. K. Burgess, D. S. DeAndrad, J. D. Marold, K. G. Fleming, Self-association of unfolded outer membrane proteins. Macromol. Biosci. 10, 763-767 (2010).

52. E. J. Danoff, K. G. Fleming, Aqueous, Unfolded OmpA forms amyloid-like fibrils upon selfassociation. PLoS One 10, e0132301 (2015).

53. L. Wang, A. Brock, B. Herberich, P. G. Schultz, Expanding the genetic code of Escherichia coli. Science (80-. ). 292, 498-500 (2001).

54. B. Hagan, J. V. Staros, Azides and nitrenes: reactivity and utility (Academic Press, 1984).

55. S. C. Reddington, et al., Different photochemical events of a genetically encoded phenyl azide define and modulate GFP fluorescence. Angew. Chemie - Int. Ed. 52, 5974-5977 (2013).

56. S. Willkomm, A. Zander, D. Grohmann, "Site-specific fluorescent labeling of argonaute for FRET-based bio-assays" in Methods in Molecular Biology, (Humana Press Inc., 2017), pp. 291-304.

57. J. W. Chin, et al., Addition of p-azido-L-phenylalanine to the genetic code of Escherichia coli. J. Am. Chem. Soc. 124, 9026-9027 (2002).

58. F. Stengel, et al., Quaternary dynamics and plasticity underlie small heat shock protein chaperone function. Proc. Natl. Acad. Sci. U. S. A. 107, 2007-12 (2010).

59. C. A. Schneider, W. S. Rasband, K. W. Eliceiri, NIH Image to ImageJ: 25 years of image analysis. Nat. Methods 9, 671-675 (2012).

60. N. R. Zaccai, et al., "Deuterium Labeling Together with Contrast Variation Small-Angle Neutron Scattering Suggests How Skp Captures and Releases Unfolded Outer Membrane Proteins" in Methods in Enzymology, (Academic Press Inc., 2016), pp. 159-210.

61. B. M. Burmann, C. Wang, S. Hiller, Conformation and dynamics of the periplasmic membrane-protein-chaperone complexes OmpX-Skp and tOmpA-Skp. Nat. Struct. Mol. Biol. 20, 1265-1272 (2013).

62. E. J. Danoff, K. G. Fleming, The soluble, periplasmic domain of OmpA folds as an 
independent unit and displays chaperone activity by reducing the self-association propensity of the unfolded OmpA transmembrane $\beta$-barrel. Biophys. Chem. 159, 194204 (2011).

63. P. J. Fleming, K. G. Fleming, HullRad: Fast Calculations of Folded and Disordered Protein and Nucleic Acid Hydrodynamic Properties. Biophys. J. 114, 856-869 (2018).

64. M. Götze, et al., Automated assignment of MS/MS cleavable cross-links in protein 3dstructure analysis. J. Am. Soc. Mass Spectrom. 26, 83-97 (2014).

65. S. El-Gebali, et al., The Pfam protein families database in 2019. Nucleic Acids Res. 47, D427-D432 (2019).

66. G. C. P. Van Zundert, et al., The HADDOCK2.2 Web Server: User-Friendly Integrative Modeling of Biomolecular Complexes. J. Mol. Biol. (2016) https:/doi.org/10.1016/j.jmb.2015.09.014.

67. J. M. A. Bullock, J. Schwab, K. Thalassinos, M. Topf, The importance of non-accessible crosslinks and solvent accessible surface distance in modeling proteins with restraints from crosslinking mass spectrometry. Mol. Cell. Proteomics 15, 2491-2500 (2016).

68. J. M. A. Bullock, K. Thalassinos, M. Topf, Jwalk and MNXL web server: model validation using restraints from crosslinking mass spectrometry. Bioinformatics 34, 3584-3585 (2018).

69. F. Pedregosa, et al., Scikit-learn: Machine Learning in Python. J. Mach. Learn. Res. 12, 2825-2830 (2011).

70. J. E. Curtis, S. Raghunandan, H. Nanda, S. Krueger, SASSIE: A program to study intrinsically disordered biological molecules and macromolecular ensembles using experimental scattering restraints. Comput. Phys. Commun. 183, 382-389 (2012).

71. J. Trewhella, et al., 2017 publication guidelines for structural modelling of small-angle scattering data from biomolecules in solution: An update. Acta Crystallogr. Sect. D Struct. Biol. (2017) https:/doi.org/10.1107/S2059798317011597.

72. T. Saio, X. Guan, P. Rossi, A. Economou, C. G. Kalodimos, Structural Basis for Protein Antiaggregation Activity of the Trigger Factor Chaperone https:/doi.org/10.1126/science.1250494 (December 4, 2019). 
73. A. J. Wirth, M. Gruebele, Quinary protein structure and the consequences of crowding in living cells: Leaving the test-tube behind. BioEssays 35, 984-993 (2013).

74. S. Wu, et al., Interaction between bacterial outer membrane proteins and periplasmic quality control factors: a kinetic partitioning mechanism. Biochem. J. 438, 505-11 (2011).

75. S. Alvira, et al., Trans-membrane association of the Sec and BAM complexes for bacterial outer-membrane biogenesis. bioRxiv https:/doi.org/10.1101/589077 (December 9, 2019).

76. D. Gessmann, et al., Outer membrane $\beta$-barrel protein folding is physically controlled by periplasmic lipid head groups and BamA. Proc. Natl. Acad. Sci. U. S. A. 111, 5878-5883 (2014).

77. M. Struyvé, M. Moons, J. Tommassen, Carboxy-terminal phenylalanine is essential for the correct assembly of a bacterial outer membrane protein. J. Mol. Biol. 218, 141-8 (1991).

78. H. De Cock, M. Struyvé, M. Kleerebezem, T. Van Der Krift, J. Tommassen, Role of the carboxy-terminal phenylalanine in the biogenesis of outer membrane protein PhoE of Escherichia coli K-12. J. Mol. Biol. 269, 473-478 (1997).

79. L. Maguire, M. Stefferson, M. D. Betterton, L. E. Hough, Design principles of selective transport through biopolymer barriers. Phys. Rev. E 100 (2019).

80. R. Wu, R. Stephenson, A. Gichaba, N. Noinaj, The big BAM theory: An open and closed case? Biochim. Biophys. Acta - Biomembr. 1862 (2020).

81. D. C. Marx, K. G. Fleming, Influence of Protein Scaffold on Side-Chain Transfer Free Energies. Biophys. J. 113, 597-604 (2017).

82. J. J. Lee, D. S. Berns, Protein aggregation. The effect of deuterium oxide on large protein aggregates of C-phycocyanin. Biochem. J. 110, 465-470 (1968).

83. B. Webb, A. Sali, Comparative protein structure modeling using MODELLER. Curr. Protoc. Bioinforma. 2016, 5.6.1-5.6.37 (2016).

84. , PyMOL. PyMOL Mol. Graph. Syst. Version 1.3 Schrödinger, LLC.

85. A. D. MacKerell, et al., All-atom empirical potential for molecular modeling and dynamics studies of proteins. J. Phys. Chem. B 102, 3586-3616 (1998).

86. J. C. Phillips, et al., Scalable molecular dynamics with NAMD. J. Comput. Chem. 26, 1781- 
bioRxiv preprint doi: https://doi.org/10.1101/2019.12.17.878660; this version posted August 13, 2020. The copyright holder for this preprint (which was not certified by peer review) is the author/funder, who has granted bioRxiv a license to display the preprint in perpetuity. It is made available under aCC-BY-NC-ND 4.0 International license.

1802 (2005).

87. G. Fiorin, M. L. Klein, J. Hénin, Using collective variables to drive molecular dynamics simulations. Mol. Phys. 111, 3345-3362 (2013). 